\title{
PERSISTENCE AND GLOBAL STABILITY IN A DELAYED PREDATOR-PREY SYSTEM WITH HOLLING-TYPE FUNCTIONAL RESPONSE
}

\author{
RUI XU ${ }^{13}$, LANSUN CHEN ${ }^{2}$ and M. A. J. CHAPLAIN ${ }^{3}$
}

(Received 19 September, 2000; revised 12 January, 2003)

\begin{abstract}
A delayed predator-prey system with Holling type III functional response is investigated. It is proved that the system is uniformly persistent under some appropriate conditions. By means of suitable Lyapunov functionals, sufficient conditions are derived for the local and global asymptotic stability of a positive equilibrium of the system. Numerical simulations are presented to illustrate the feasibility of our main results.
\end{abstract}

\section{Introduction}

The functional response is a key element in all predator-prey interactions. In population dynamics, the functional response refers to the number of prey eaten per predator per unit time as a function of prey density. Holling $[19,20]$ studied predation of small mammals on pine sawflies and found that predation rates increased with increasing prey population density. This resulted from two effects: (1) each predator increased its consumption rate when exposed to a higher prey density, and (2) predator density increased with increasing prey density. Holling suggested three kinds of functional responses as follows:

$$
\text { (1) } p_{1}(x)=a x, \quad \text { (2) } \quad p_{2}(x)=\frac{a x}{m+x}, \quad \text { (3) } p_{3}(x)=\frac{a x^{2}}{m+x^{2}},
$$

where $x$ represents the density of prey. Functions $p_{1}(x), p_{2}(x)$ and $p_{3}(x)$ are now referred to as Holling type I, II and II functional responses. Function $p_{2}(x)$ is also

\footnotetext{
${ }^{1}$ Department of Mathematics, Shijiazhuang Mechanical Engineering College, Shijiazhuang 050003, Hebei Province, P.R. China; e-mail: rxu88@yahoo.com.cn.

${ }^{2}$ Institute of Mathematics, Academia Sinica, Beijing 100080, P.R. China.

${ }^{3}$ Department of Mathematics, University of Dundee, Dundee DDI 4HN, Scotland, U.K.

(C) Australian Mathematical Society 2004, Serial-fee code 1446-1811/04
} 
referred to as a Michaelis-Menten function in studying enzymatic reactions. Holling type II and III responses illustrate the principle of time budgets in behavioural ecology. This principle assumes that a predator spends its time on two kinds of activities: searching for prey and prey handling which includes: chasing, killing, eating and digesting.

Type I functional responses (linear) are found in passive predators like spiders. The number of flies caught in a net is proportional to fly density. Prey mortality due to predation is constant.

Type II functional responses are most typical of predators that specialise in one or a few prey. Here $a>0$ denotes the search rate of the predator and $m>0$ is the half-saturation constant. Prey mortality declines with prey density. Predators of this type cause maximum mortality at low prey density. For example, small mammals destroy most gypsy moth pupae in sparse populations of gypsy moths. However in high-density defoliating populations, small mammals kill a negligible proportion of pupae.

In type III functional responses (sigmoid), the risk of being preyed upon is small at low prey density but increases up to a certain point as prey density increases. This is referred to as positive density-dependent or stabilising mortality (Hassell [12], Holling [21]). Several factors can lead to a type III functional response such as predator learning, prey refuge and the presence of alternative prey (Holling [21]). The presence of a prey refuge has been hypothesised to be a factor leading to positive densitydependent mortality in several predator-prey systems (Bailey [1], Hassell [12], Hixon and Carr [17]). Alternative prey can lead to a type III functional response through switching behaviour (Murdoch [31], Murdoch et al. [32], Murdoch and Marks [33]).

Predator-prey systems with Holling-type functional responses have been studied extensively and the dynamics of such systems are now very well understood. The analysis of these models has been centred around the persistence of populations, the stability of equilibria, the existence and uniqueness of limit cycles, and Hopf bifurcations (see, for example, $[2,3,6,11,16,18,23,24,27,29,36]$ and the references cited therein).

We note that time delays of one type or another have been incorporated into biological models by many researchers; we refer to the monographs of Cushing [5], Gopalsamy [10], Kuang [25] and MacDonald [28] for general delayed biological systems and to Beretta and Kuang [4], Gopalsamy [8, 9], Hastings [13], He [14, 15], May [30], Ruan [34], Wang and Ma [37], Xu and Yang [39], and the references cited therein for studies on delayed biological systems. In general, delay differential equations exhibit much more complicated dynamics than ordinary differential equations since a time delay could cause a stable equilibrium to become unstable and cause the population to fluctuate. Time delay due to gestation is a common example, because generally the consumption of prey by a predator throughout its past history governs 
the present birth rate of the predator. Therefore more realistic models of population interactions should take into account the effect of time delays.

An important problem in predator-prey theory and related topics in mathematical ecology concerns the global stability of an ecological system with time delays. However, most of the global stability or convergence results appearing so far for delayed ecological systems require that the instantaneous negative feedbacks dominate both delayed feedback and interspecific interactions. Such a requirement is rarely met in real systems since feedbacks are generally delayed. This leads to the standing question: under what conditions will the global stability of a nonnegative steady state of a delay differential system persist when time delays involved in some part of the negative feedbacks are small enough? Kuang [26] presented a partial answer to this open question for Lotka-Volterra-type systems.

The objective of this paper is to study the combined effects of functional response and time delays on the dynamics of predator-prey systems. Motivated by the work of Kuang [26], we consider the following delayed Holling type III predator-prey system without dominating instantaneous negative feedback:

$$
\left\{\begin{array}{l}
\dot{x}_{1}(t)=x_{1}(t)\left(a_{1}-a_{11} x_{1}\left(t-\tau_{1}\right)-a_{12} \frac{x_{1}(t) x_{2}(t)}{m+x_{1}^{2}(t)}\right), \\
\dot{x}_{2}(t)=x_{2}(t)\left(-a_{2}+a_{21} \frac{x_{1}^{2}\left(t-\tau_{2}\right)}{m+x_{1}^{2}\left(t-\tau_{2}\right)}-a_{22} x_{2}\left(t-\tau_{3}\right)\right)
\end{array}\right.
$$

with initial conditions

$$
\begin{gathered}
x_{i}(\theta)=\phi_{i}(\theta), \quad \theta \in[-\tau, 0], \quad \phi_{i}(0)>0, \\
\phi_{i} \in C\left([-\tau, 0), R_{+}\right), \quad i=1,2,
\end{gathered}
$$

where $x_{1}(t), x_{2}(t)$ denote the densities of the prey and the predator at time $t$, respectively. Here $a_{i}, a_{i j}(i, j=1,2)$ are positive constants, $\tau_{i}(i=1,2,3)$ are nonnegative constants and $\tau=\max \left\{\tau_{1}, \tau_{2}, \tau_{3}\right\}$. Also $a_{1}$ is the intrinsic growth rate of the prey, $a_{11}$ is the intra-specific competition rate of the prey, $a_{1} / a_{11}$ is the carrying capacity of the prey, $a_{12}$ is the capturing rate of the predator, $m$ is the half capturing saturation constant, $a_{21} / a_{12}$ is the rate of conversion of nutrients into the reproduction of the predator and $a_{2}$ is the death rate of the predator. We note that $\tau_{1} \geq 0$ denotes the delay in the negative feedback of the prey species and that $\tau_{2}$ is the delay due to gestation, that is, mature adult predators can only contribute to the reproduction of the predator biomass. In addition, we have included the term $-a_{22} x_{2}\left(t-\tau_{3}\right)$ in the dynamics of the predator to incorporate the negative feedback of predator crowding.

We adopt the following notation and concepts throughout this paper.

Let $R_{+}^{2}=\left\{x \in R^{2}: x_{1} \geq 0, x_{2} \geq 0\right\}$. For ecological reasons, we consider system (1.1) only in Int $R_{+}^{2}$. 
DEFINITION 1.1. System (1.1) is said to be uniformly persistent if there exists a compact region $D \subset$ Int $R_{+}^{2}$ such that every solution $x(t)=\left(x_{1}(t), x_{2}(t)\right)$ of system (1.1) with initial conditions (1.2) eventually enters and remains in the region $D$.

The organisation of this paper is as follows. In the next section, we present a permanence result for system (1.1). In Section 3, we establish conditions for the local stability of a positive equilibrium of system (1.1) and show that these conditions depend on $\tau_{1}$ and $\tau_{3}$. In Section 4 , sufficient conditions are derived for the global asymptotic stability of the positive equilibrium of system (1.1). Some examples and numerical simulations are presented in Section 5 to illustrate the feasibility of our main results. In Section 6, a brief discussion is given to conclude this work.

\section{Uniform persistence}

In this section, by using the criterion proposed by Freedman and Ruan [7] for retarded functional differential equations, we establish sufficient conditions to guarantee the persistence of system (1.1). The following lemmas are elementary and are concerned with the qualitative nature of solutions of system (1.1).

LEMMA 2.1. Solutions of system (1.1) with initial conditions (1.2) are defined on $[0,+\infty)$ and remain positive for all $t \geq 0$.

LEMMA 2.2. Let $x(t)=\left(x_{1}(t), x_{2}(t)\right)$ denote any positive solution of system (1.1) with initial conditions (1.2). Then there exists a $T>0$ such that if $t \geq T$

$$
x_{1}(t) \leq M_{1}, \quad x_{2}(t) \leq M_{2},
$$

where

$$
M_{1}=\frac{a_{1}}{a_{11}} e^{a_{1} \tau_{1}}, \quad M_{2}=\frac{a_{21}}{a_{22}} e^{a_{21} \tau_{3}} .
$$

The proofs of Lemmas 2.1 and 2.2 are similar to those of Lemmas 2.1 and 2.2 of [37] and we therefore omit them here.

We are now in a position to establish the uniform persistence of system (1.1).

THEOREM 2.1. System (1.1) is uniformly persistent provided that

(H1) $a_{1}^{2}\left(a_{21}-a_{2}\right)>m a_{2} a_{11}^{2}$,

(H2) $\quad a_{1} \tau_{1} \leq 3 / 2$.

PROOF. It is easy to verify that system $(1.1)$ has two equilibria $E_{0}(0,0)$ and $E_{1}\left(a_{1} / a_{11}, 0\right)$ on the boundary of $R_{+}^{2}$. From the assumptions of the theorem we know that the omega limit set of the boundary of $R_{+}^{2}$ is the union of the boundary 
equilibria $E_{0}$ and $E_{1}$. We choose $p\left(x_{1}(t), x_{2}(t)\right)=x_{1}^{\alpha_{1}}(t) x_{2}^{\alpha_{2}}(t)$, where $\alpha_{i}(i=1,2)$ are undetermined positive constants. We have

$$
\begin{aligned}
\psi(x)=\frac{\dot{p}(x)}{p(x)}= & \alpha_{1}\left(a_{1}-a_{11} x_{1}\left(t-\tau_{1}\right)-a_{12} \frac{x_{1}(t) x_{2}(t)}{m+x_{1}^{2}(t)}\right) \\
& +\alpha_{2}\left(-a_{2}+a_{21} \frac{x_{1}^{2}\left(t-\tau_{2}\right)}{m+x_{1}^{2}\left(t-\tau_{2}\right)}-a_{22} x_{2}\left(t-\tau_{3}\right)\right) .
\end{aligned}
$$

If we choose $\alpha_{1}=1$, and $\alpha_{2}$ so small that $\alpha_{1} a_{1}-\alpha_{2} a_{2}>0$, then $\psi$ is positive at $E_{0}$. Under Assumption ( $\mathrm{H} 1)$, it is easy to verify that $\psi$ is positive at $E_{1}$. Hence there is a choice of $\alpha_{2}$ to ensure $\psi>0$ at the boundary equilibria. If the condition (H2) holds, it follows from [38] that $E_{1}$ is globally asymptotically stable with respect to solutions initiating in the $x_{1}$-axis. It is easy to verify that $E_{0}$ is globally asymptotically stable in the $x_{2}$-axis. Thus it follows from Theorem 3.12 of Freedman and Ruan [7] that system (1.1) is uniformly persistent.

REMARK 1. If $\tau_{1}=\tau_{2}=\tau_{3}=0$, then system (1.1) reduces to an instantaneous system, that is, one without time delay. From the proof of Theorem 2.1; we see that if (H1) holds, then the corresponding instantaneous system of (1.1) is uniformly persistent, which implies that system (1.1) must have at least one positive equilibrium (see Hutson [22]).

\section{Local asymptotic stability}

From Section 2, we see that if (H1) holds, then system (1.1) has at least one positive equilibrium. Let $E^{*}\left(x_{1}^{*}, x_{2}^{*}\right)$ be a positive equilibrium of system (1.1). In this section, we discuss the local asymptotic stability of the positive equilibrium $E^{*}\left(x_{1}^{*}, x_{2}^{*}\right)$.

Linearising system $(1.1)$ at $E^{*}\left(x_{1}^{*}, x_{2}^{*}\right)$, we obtain

$$
\left\{\begin{array}{l}
\dot{N}_{1}(t)=A_{11} N_{1}\left(t-\tau_{1}\right)+B_{11} N_{1}(t)+A_{12} N_{2}(t), \\
\dot{N}_{2}(t)=A_{21} N_{1}\left(t-\tau_{2}\right)+A_{22} N_{2}\left(t-\tau_{3}\right)
\end{array}\right.
$$

where

$$
\begin{array}{ll}
A_{11}=-a_{11} x_{1}^{*}, & A_{12}=-\frac{a_{12} x_{1}^{* 2}}{m+x_{1}^{* 2}}, \quad B_{11}=\frac{a_{12} x_{1}^{*} x_{2}^{*}\left(x_{1}^{* 2}-m\right)}{\left(m+x_{1}^{* 2}\right)^{2}}, \\
A_{21}=\frac{2 m a_{21} x_{1}^{*} x_{2}^{*}}{\left(m+x_{1}^{* 2}\right)^{2}}, & A_{22}=-a_{22} x_{2}^{*} .
\end{array}
$$

We note that the locally uniformly asymptotic stability of the positive equilibrium $E^{*}\left(x_{1}^{*}, x_{2}^{*}\right)$ of system (1.1) follows from that of the zero solution of system (3.1) (see Kuang [25, Theorem 4.2, page 26]). 
THEOREM 3.1. Let $(\mathrm{H} 1)$ hold. Assume further that

(H3) $A_{11} \tau_{1}\left(2 A_{11}-2\left|B_{11}\right|+A_{12}\right)-A_{21} A_{22} \tau_{3}<-2\left(A_{11}+B_{11}\right)+A_{12}-A_{21}$,

(H4) $A_{11} A_{12} \tau_{1}-A_{22} \tau_{3}\left(A_{21}-2 A_{22}\right)<-2 A_{22}+A_{12}-A_{21}$.

Then the positive equilibrium $E^{*}$ of system (1.1) is uniformly asymptotically stable.

PROOF. The first equation of (3.1) can be rewritten as

$$
\begin{aligned}
\dot{N}_{1}(t)= & \left(A_{11}+B_{11}\right) N_{1}(t)+A_{12} N_{2}(t)-A_{11} \int_{t-\tau_{1}}^{t} \dot{N}_{1}(u) d u \\
= & \left(A_{11}+B_{11}\right) N_{1}(t)+A_{12} N_{2}(t) \\
& -A_{11} \int_{t-\tau_{1}}^{t}\left(A_{11} N_{1}\left(u-\tau_{1}\right)+B_{11} N_{1}(u)+A_{12} N_{2}(u)\right) d u .
\end{aligned}
$$

Define $W_{11}(t)=N_{1}^{2}(t)$. Calculating the derivative of $W_{11}(t)$ along solutions of (3.1), we have

$$
\begin{aligned}
\frac{d}{d t} W_{11}(t)= & 2 N_{1}(t)\left\{\left(A_{11}+B_{11}\right) N_{1}(t)+A_{12} N_{2}(t)\right. \\
& \left.-A_{11} \int_{t-\tau_{1}}^{t}\left(A_{11} N_{1}\left(u-\tau_{1}\right)+B_{11} N_{1}(u)+A_{12} N_{2}(u)\right) d u\right\} \\
= & 2\left(A_{11}+B_{11}\right) N_{1}^{2}(t)+2 A_{12} N_{1}(t) N_{2}(t) \\
& -2 A_{11} N_{1}(t) \int_{t-\tau_{1}}^{t}\left(A_{11} N_{1}\left(u-\tau_{1}\right)+B_{11} N_{1}(u)+A_{12} N_{2}(u)\right) d u .
\end{aligned}
$$

Using the inequality $a^{2}+b^{2} \geq 2 a b$, we get

$$
\begin{aligned}
\frac{d}{d t} W_{11}(t) \leq & 2\left(A_{11}+B_{11}\right) N_{1}^{2}(t)-A_{12} N_{1}^{2}(t)-A_{12} N_{2}^{2}(t) \\
& +A_{11} \tau_{1}\left(A_{11}-\left|B_{11}\right|+A_{12}\right) N_{1}^{2}(t) \\
& +A_{11} \int_{t-\tau_{1}}^{t}\left[A_{11} N_{1}^{2}\left(u-\tau_{1}\right)-\left|B_{11}\right| N_{1}^{2}(u)+A_{12} N_{2}^{2}(u)\right] d u .
\end{aligned}
$$

Define $W_{12}(t)$ as

$$
W_{12}(t)=A_{11} \int_{t-\tau_{1}}^{t} \int_{v}^{t}\left[A_{11} N_{1}^{2}\left(u-\tau_{1}\right)-\left|B_{11}\right| N_{1}^{2}(u)+A_{12} N_{2}^{2}(u)\right] d u d v
$$

It follows from (3.2) and (3.3) that

$$
\begin{aligned}
\frac{d}{d t}\left(W_{11}(t)+W_{12}(t)\right) & \\
\leq & {\left[2\left(A_{11}+B_{11}\right)-A_{12}+A_{11} \tau_{1}\left(A_{11}-\left|B_{11}\right|+A_{12}\right)\right] N_{1}^{2}(t) } \\
& -A_{12} N_{2}^{2}(t)+A_{11} \tau_{1}\left[A_{11} N_{1}^{2}\left(t-\tau_{1}\right)-\left|B_{11}\right| N_{1}^{2}(t)+A_{12} N_{2}^{2}(t)\right] .
\end{aligned}
$$


Let $W_{1}(t)$ be defined by

$$
W_{1}(t)=W_{11}(t)+W_{12}(t)+W_{13}(t)
$$

where

$$
W_{13}(t)=A_{11}^{2} \tau_{1} \int_{t-\tau_{1}}^{t} N_{1}^{2}(u) d u .
$$

Then we derive from (3.4)-(3.6) that

$$
\begin{aligned}
\frac{d}{d t} W_{1}(t) \leq & {\left[2\left(A_{11}+B_{11}\right)-A_{12}+\tau_{1} A_{11}\left(A_{11}-\left|B_{11}\right|+A_{12}\right)\right] N_{1}^{2}(t) } \\
& -A_{12} N_{2}^{2}(t)+A_{11} \tau_{1}\left[A_{11} N_{1}^{2}(t)-\left|B_{11}\right| N_{1}^{2}(t)+A_{12} N_{2}^{2}(t)\right] \\
= & {\left[2\left(A_{11}+B_{11}\right)-A_{12}+\tau_{1} A_{11}\left(2 A_{11}-2\left|B_{11}\right|+A_{12}\right)\right] N_{1}^{2}(t) } \\
& -A_{12}\left(1-A_{11} \tau_{1}\right) N_{2}^{2}(t) .
\end{aligned}
$$

Similarly, the second equation of (3.1) can be rewritten as

$$
\begin{aligned}
\dot{N}_{2}(t) & =A_{22} N_{2}(t)+A_{21} N_{1}\left(t-\tau_{2}\right)-A_{22} \int_{t-\tau_{3}}^{t} \dot{N}_{2}(u) d u \\
& =A_{22} N_{2}(t)+A_{21} N_{1}\left(t-\tau_{2}\right)-A_{22} \int_{t-\tau_{3}}^{t}\left[A_{21} N_{1}\left(u-\tau_{2}\right)+A_{22} N_{2}\left(u-\tau_{3}\right)\right] d u .
\end{aligned}
$$

We define $W_{21}(t)=N_{2}^{2}(t)$. Then calculating the derivative of $W_{21}(t)$ along solutions of (3.1), we derive that

$$
\begin{aligned}
\frac{d}{d t} W_{21}(t)= & 2 N_{2}(t)\left\{A_{22} N_{2}(t)+A_{21} N_{1}\left(t-\tau_{2}\right)\right. \\
& \left.-A_{22} \int_{t-\tau_{3}}^{t}\left[A_{21} N_{1}\left(u-\tau_{2}\right)+A_{22} N_{2}\left(u-\tau_{3}\right)\right] d u\right\} \\
= & 2 A_{22} N_{2}^{2}(t)+2 A_{21} N_{1}\left(t-\tau_{2}\right) N_{2}(t) \\
& -2 A_{22} N_{2}(t) \int_{t-\tau_{3}}^{t}\left[A_{21} N_{1}\left(u-\tau_{2}\right)+A_{22} N_{2}\left(u-\tau_{3}\right)\right] d u .
\end{aligned}
$$

Using the inequality $a^{2}+b^{2} \geq 2 a b$, we get

$$
\begin{aligned}
\frac{d}{d t} W_{21}(t) \leq & 2 A_{22} N_{2}^{2}(t)+A_{21} N_{1}^{2}\left(t-\tau_{2}\right) \\
& +A_{21} N_{2}^{2}(t)-A_{22} \tau_{3}\left(A_{21}-A_{22}\right) N_{2}^{2}(t) \\
& -A_{22} \int_{t-\tau_{3}}^{t}\left[A_{21} N_{1}^{2}\left(u-\tau_{2}\right)-A_{22} N_{2}^{2}\left(u-\tau_{3}\right)\right] d u
\end{aligned}
$$


Define $W_{22}(t)$ as

$$
\begin{aligned}
W_{22}(t)= & -A_{22} \int_{t-\tau_{3}}^{t} \int_{v}^{t}\left[A_{21} N_{1}^{2}\left(u-\tau_{2}\right)-A_{22} N_{2}^{2}\left(u-\tau_{3}\right)\right] d u d v \\
& +A_{21} \int_{t-\tau_{2}}^{t} N_{1}^{2}(u) d u .
\end{aligned}
$$

It follows from (3.7) and (3.8) that

$$
\begin{aligned}
\frac{d}{d t}\left(W_{21}(t)+W_{22}(t)\right) \leq & {\left[2 A_{22}+A_{21}-A_{22} \tau_{3}\left(A_{21}-A_{22}\right)\right] N_{2}^{2}(t)+A_{21} N_{1}^{2}(t) } \\
& -A_{22} \tau_{3}\left[A_{21} N_{1}^{2}\left(t-\tau_{2}\right)-A_{22} N_{2}^{2}\left(t-\tau_{3}\right)\right]
\end{aligned}
$$

Let $W_{2}(t)$ be defined by

$$
W_{2}(t)=W_{21}(t)+W_{22}(t)+W_{23}(t)
$$

where

$$
W_{23}(t)=-A_{22} \tau_{3}\left(A_{21} \int_{t-\tau_{2}}^{t} N_{1}^{2}(u) d u-A_{22} \int_{t-\tau_{3}}^{t} N_{2}^{2}(u) d u\right) .
$$

Then we derive from (3.9)-(3.11) that

$$
\begin{aligned}
\frac{d}{d t} W_{2}(t) \leq & {\left[2 A_{22}+A_{21}-A_{22} \tau_{3}\left(A_{21}-A_{22}\right)\right] N_{2}^{2}(t)+A_{21} N_{1}^{2}(t) } \\
& -A_{22} \tau_{3}\left[A_{21} N_{1}^{2}(t)-A_{22} N_{2}^{2}(t)\right] \\
= & {\left[2 A_{22}+A_{21}-A_{22} \tau_{3}\left(A_{21}-2 A_{22}\right)\right] N_{2}^{2}(t)+A_{21}\left(1-A_{22} \tau_{3}\right) N_{1}^{2}(t) . }
\end{aligned}
$$

Let $W(t)=W_{1}(t)+W_{2}(t)$. Then calculating the derivative of $W(t)$ along solutions of (3.1), we have

$$
\begin{aligned}
\frac{d}{d t} W(t) \leq & {\left[2\left(A_{11}+B_{11}\right)-A_{12}+A_{11} \tau_{1}\left(2 A_{11}-2\left|B_{11}\right|+A_{12}\right)\right] N_{1}^{2}(t) } \\
& -A_{12}\left(1-A_{11} \tau_{1}\right) N_{2}^{2}(t)+A_{21}\left(1-A_{22} \tau_{3}\right) N_{1}^{2}(t) \\
& +\left[2 A_{22}+A_{21}-A_{22} \tau_{3}\left(A_{21}-2 A_{22}\right)\right] N_{2}^{2}(t) \\
= & -\alpha_{1} N_{1}^{2}(t)-\alpha_{2} N_{2}^{2}(t)
\end{aligned}
$$

where

$$
\begin{aligned}
& \alpha_{1}=-\left[2\left(A_{11}+B_{11}\right)-A_{12}+A_{21}+A_{11} \tau_{1}\left(2 A_{11}-2\left|B_{11}\right|+A_{12}\right)-A_{21} A_{22} \tau_{3}\right], \\
& \alpha_{2}=-\left[2 A_{22}-A_{12}+A_{21}+A_{11} A_{12} \tau_{1}-A_{22} \tau_{3}\left(A_{21}-2 A_{22}\right)\right] .
\end{aligned}
$$

Clearly, Assumptions (H3)-(H4) imply that $\alpha_{1}>0, \alpha_{2}>0$. According to the Lyapunov theorem (see Kuang [25, Theorem 5.1, page 27]), we see that the zero solution of (3.1) is uniformly asymptotically stable. Accordingly, the positive equilibrium $E^{*}$ of system (1.1) is uniformly asymptotically stable. 
COROLlaRY 3.1. Let $(\mathrm{H1})$ hold. Assume further that $2\left(A_{11}+B_{11}\right)-A_{12}+A_{21}<0$, $2 A_{22}-A_{12}+A_{21}<0$. Then the positive equilibrium $E^{*}\left(x_{1}^{*}, x_{2}^{*}\right)$ is locally asymptotically stable provided that $0 \leq \max \left\{\tau_{1}, \tau_{3}\right\} \leq \tau_{L}$, where

$$
\tau_{L}=\min \left\{\frac{-2\left(A_{11}+B_{11}\right)+A_{12}-A_{21}}{A_{11}\left(2 A_{11}-2\left|B_{11}\right|+A_{12}\right)-A_{21} A_{22}}, \frac{-2 A_{22}+A_{12}-A_{21}}{A_{11} A_{12}-A_{22}\left(A_{21}-2 A_{22}\right)}\right\} .
$$

REMARK 2. By using the Lyapunov function $V(t)=\left(-A_{21} / A_{12}\right) N_{1}^{2}(t)+N_{2}^{2}(t)$ one can easily prove that if $A_{11}+B_{11}<0$, then the positive equilibrium of the "instantaneous" (when $\tau_{i}=0, i=1,2,3$ ) model (1.1) is locally uniformly asymptotically stable. If $2\left(A_{11}+B_{11}\right)-A_{12}+A_{21}<0$ and $2 A_{22}-A_{12}+A_{21}<0$, then the local uniform asymptotic stability of $E^{*}$ of the delayed model (1.1) is preserved for small $\tau_{1}$ and $\tau_{3}$ satisfying $(\mathrm{H} 3)-(\mathrm{H} 4)$.

It is interesting to discuss the effect of time delays on the stability of the positive equilibrium of (1.1). We assume that the positive equilibrium $E^{*}$ exists for system (1.1). For simplicity, first, we let $\tau_{1}=\tau_{3}=0, \tau_{2}=\tau$.

The characteristic equation for (3.1) takes the form

$$
P_{1}(\lambda)+Q_{1}(\lambda) e^{-\lambda \tau}=0,
$$

where $P_{1}(\lambda)=\left(\lambda-A_{11}-B_{11}\right)\left(\lambda-A_{22}\right)$ and $Q_{1}(\lambda)=-A_{12} A_{21}$. If $A_{11}+B_{11}<0$, then from the discussions in Remark 2 we see that the positive equilibrium $E^{*}$ of (1.1) is stable for $\tau=0$. It is easy to examine that

$$
\begin{aligned}
F_{1}(y) & =\left|P_{1}(i y)\right|^{2}-\left|Q_{1}(i y)\right|^{2} \\
& =y^{4}+\left[\left(A_{11}+B_{11}\right)^{2}+A_{22}^{2}\right] y^{2}+A_{22}^{2}\left(A_{11}+B_{11}\right)^{2}-A_{12}^{2} A_{21}^{2} .
\end{aligned}
$$

If $A_{11}+B_{11}<0$ and $A_{22}\left(A_{11}+B_{11}\right)+A_{12} A_{21}>0$, then it is easy to verify that $F_{1}(y)=0$ has no positive roots; if $A_{11}+B_{11}<0$ and $A_{22}\left(A_{11}+B_{11}\right)+A_{12} A_{21}<0$, then $F_{1}(y)=0$ has a unique positive root. By applying [25, Theorem 4.1, page 83], we see that if $A_{11}+B_{11}<0$ and $A_{22}\left(A_{11}+B_{11}\right)+A_{12} A_{21}>0$, as $\tau$ increases, no stability switch may occur; if $A_{11}+B_{11}<0$ and $A_{22}\left(A_{11}+B_{11}\right)+A_{12} A_{21}<0$, then there is a positive constant $\tau_{0}$ (which can be evaluated explicitly) such that for $\tau>\tau_{0}, E^{*}$ becomes unstable. Notice that if $2\left(A_{11}+B_{11}\right)-A_{12}+A_{21}<0,2 A_{22}-A_{12}+A_{21}<0$, then it is easy to verify that $A_{11}+B_{11}<0, A_{22}\left(A_{11}+B_{11}\right)+A_{12} A_{21}>0$. Thus, in this case, from the discussions above, we see that the delay due to gestation of the predator is harmless for the local stability of the positive equilibrium $E^{*}$ of system (1.1).

Secondly, we set $\tau_{1}=\tau_{2}=0, \tau_{3}=\tau$. Then the characteristic equation for (3.1) takes the form

$$
P_{2}(\lambda)+Q_{2}(\lambda) e^{-\lambda \tau}=0
$$


where $P_{2}(\lambda)=\lambda\left(\lambda-A_{11}-B_{11}\right)-A_{12} A_{21}, Q_{2}(\lambda)=-A_{22}\left(\lambda-A_{11}-B_{11}\right)$. It is easy to demonstrate that

$$
\begin{aligned}
F_{2}(y) & =\left|P_{2}(i y)\right|^{2}-\left|Q_{2}(i y)\right|^{2} \\
& =y^{4}+\left[\left(A_{11}+B_{11}\right)^{2}-A_{22}^{2}+2 A_{12} A_{21}\right] y^{2}+A_{12}^{2} A_{21}^{2}-A_{22}^{2}\left(A_{11}+B_{11}\right)^{2} .
\end{aligned}
$$

Set

$$
\Delta=\left[\left(A_{11}+B_{11}\right)^{2}-A_{22}^{2}+2 A_{12} A_{21}\right]^{2}-4\left[A_{12}^{2} A_{21}^{2}-A_{22}^{2}\left(A_{11}+B_{11}\right)^{2}\right] .
$$

If $\Delta<0$ or $\Delta \geq 0, A_{11}+B_{11}<0, A_{22}\left(A_{11}+B_{11}\right)+A_{12} A_{21}<0$ and $\left(A_{11}+B_{11}\right)^{2}-$ $A_{22}^{2}+2 A_{12} A_{21}>0$, then $F_{2}(y)=0$ has no positive roots. In this case, as $\tau$ increases, no stability switch may occur; if $A_{11}+B_{11}<0$ and $A_{22}\left(A_{11}+B_{11}\right)+A_{12} A_{21}>0$, then it is easy to verify that $F_{2}(y)=0$ has a unique positive root which is simple. Accordingly, there is a positive constant $\tau_{0}$, such that for $\tau>\tau_{0}, E^{*}$ becomes unstable.

If we let $\tau_{2}=\tau_{3}=0, \tau_{1}=\tau$, a similar conclusion can be obtained for (1.1). Therefore if $A_{11}+B_{11}<0$ and $A_{22}\left(A_{11}+B_{11}\right)+A_{12} A_{21}>0$, then time delays due to negative feedbacks of the prey and predator destabilise $E^{*}$ for (1.1). So does the delay due to gestation of the predator if $A_{11}+B_{11}<0$ and $A_{22}\left(A_{11}+B_{11}\right)+A_{12} A_{21}<0$.

\section{Global asymptotic stability}

In this section, we provide conditions under which the positive equilibrium $E^{*}$ of system (1.1) is globally asymptotically stable. The method of proof is to construct a suitable Lyapunov functional for system (1.1) by borrowing the technique used in $[14,15]$. It is immediate that if the conditions for the global stability of the positive equilibrium $E^{*}\left(x_{1}^{*}, x_{2}^{*}\right)$ are explicitly independent of $x_{1}^{*}$ and $x_{2}^{*}$, then $E^{*}\left(x_{1}^{*}, x_{2}^{*}\right)$ is in fact unique.

THEOREM 4.1. Suppose that system (1.1) sati.fies (H1)-(H2). Then the positive equilibrium $E^{*}$ of system (1.1) is globally asymptotically stable provided that

(H5) $r_{i i}>0, i=1,2$,

(H6) $r_{11} r_{22}-r_{12} r_{21}>0$,

where

$$
\begin{gathered}
r_{11}=2 a_{11}-\frac{a_{1}}{2 \sqrt{m}}-a_{1} \sqrt{\frac{a_{21}-a_{2}}{m a_{2}}}-a_{11} M_{1} \tau_{1}\left(\frac{a_{1}}{2 \sqrt{m}}+a_{1} \sqrt{\frac{a_{21}-a_{2}}{m a_{2}}}\right), \\
r_{12}=-\frac{a_{12}}{2 \sqrt{m}}\left(1+a_{11} M_{1} \tau_{1}\right), \quad r_{21}=-\frac{a_{21}}{\sqrt{m}}\left(1+a_{22} M_{2} \tau_{3}\right), \quad r_{22}=a_{22}\left(1-a_{22} M_{2} \tau_{3}\right),
\end{gathered}
$$

in which $M_{1}$ and $M_{2}$ are defined by (2.2). 
PROOF. Let $x(t)=\left(x_{1}(t), x_{2}(t)\right)$ be any solution of (1.1) with initial conditions (1.2). Define $u(t)=\left(u_{1}(t), u_{2}(t)\right)$ by

$$
u_{1}(t)=\ln \frac{x_{1}(t)}{x_{1}^{*}} \quad \text { and } \quad u_{2}(t)=\ln \frac{x_{2}(t)}{x_{2}^{*}} .
$$

On substituting (4.1) into (1.1), we derive

$$
\left\{\begin{aligned}
\frac{d u_{1}}{d t}= & -a_{11} x_{1}^{*}\left(e^{u_{1}\left(t-\tau_{1}\right)}-1\right)-\frac{a_{12} x_{2}^{*} x_{1}}{m+x_{1}^{2}}\left(e^{u_{2}(t)}-1\right) \\
& +\frac{a_{12} x_{1}^{*} x_{2}^{*}\left(x_{1}^{*} x_{1}-m\right)}{\left(m+x_{1}^{2}\right)\left(m+x_{1}^{* 2}\right)}\left(e^{u_{1}(t)}-1\right) \\
\frac{d u_{2}}{d t}= & \frac{m a_{21} x_{1}^{*}\left(x_{1}\left(t-\tau_{2}\right)+x_{1}^{*}\right)}{\left(m+x_{1}^{2}\left(t-\tau_{2}\right)\right)\left(m+x_{1}^{* 2}\right)}\left(e^{u_{1}\left(t-\tau_{2}\right)}-1\right) \\
& -a_{22} x_{2}^{*}\left(e^{u_{2}\left(t-\tau_{3}\right)}-1\right) .
\end{aligned}\right.
$$

The first equation of (4.2) can be rewritten as

$$
\begin{aligned}
\frac{d u_{1}}{d t}= & -a_{11} x_{1}^{*}\left(e^{u_{1}(t)}-1\right)-\frac{a_{12} x_{2}^{*} x_{1}}{m+x_{1}^{2}}\left(e^{u_{2}(t)}-1\right) \\
& +\frac{a_{12} x_{1}^{*} x_{2}^{*}\left(x_{1}^{*} x_{1}-m\right)}{\left(m+x_{1}^{2}\right)\left(m+x_{1}^{* 2}\right)}\left(e^{u_{1}(t)}-1\right)+a_{11} x_{1}^{*} \int_{t-\tau_{1}}^{t} e^{u_{1}(s)} \frac{d u_{1}(s)}{d s} d s \\
= & -a_{11} x_{1}^{*}\left(e^{u_{1}(t)}-1\right)-\frac{a_{12} x_{2}^{*} x_{1}}{m+x_{1}^{2}}\left(e^{u_{2}(t)}-1\right) \\
& +\frac{a_{12} x_{1}^{*} x_{2}^{*}\left(x_{1}^{*} x_{1}-m\right)}{\left(m+x_{1}^{2}\right)\left(m+x_{1}^{* 2}\right)}\left(e^{u_{1}(t)}-1\right) \\
& +a_{11} x_{1}^{*} \int_{t-\tau_{1}}^{t} e^{u_{1}(s)}\left\{-a_{11} x_{1}^{*}\left(e^{u_{1}\left(s-\tau_{1}\right)}-1\right)-\frac{a_{12} x_{2}^{*} x_{1}}{m+x_{1}^{2}}\left(e^{u_{2}(s)}-1\right)\right. \\
& \left.+\frac{a_{12} x_{1}^{*} x_{2}^{*}\left(x_{1}^{*} x_{1}-m\right)}{\left(m+x_{1}^{2}\right)\left(m+x_{1}^{* 2}\right)}\left(e^{u_{1}(s)}-1\right)\right\} d s .
\end{aligned}
$$

Let

$$
V_{11}(t)=\left|u_{1}(t)\right|
$$

Calculating the upper right derivative of $V_{11}(t)$ along solutions of (4.2), it follows from (4.3) and (4.4) that

$$
\begin{aligned}
D^{+} V_{11}(t) \leq & -a_{11} x_{1}^{*}\left|e^{u_{1}(t)}-1\right|+\frac{a_{12} x_{2}^{*}}{2 \sqrt{m}}\left|e^{u_{2}(t)}-1\right| \\
& +\frac{a_{12} x_{1}^{*} x_{2}^{*}\left(x_{1}^{*}+2 \sqrt{m}\right)}{2 \sqrt{m}\left(m+x_{1}^{* 2}\right)}\left|e^{u_{1}(t)}-1\right|
\end{aligned}
$$




$$
\begin{aligned}
& +a_{11} x_{1}^{*} \int_{t-\tau_{1}}^{t} e^{u_{1}(s)}\left\{a_{11} x_{1}^{*}\left|e^{u_{1}\left(s-\tau_{1}\right)}-1\right|+\frac{a_{12} x_{2}^{*}}{2 \sqrt{m}}\left|e^{u_{2}(s)}-1\right|\right. \\
& \left.+\frac{a_{12} x_{1}^{*} x_{2}^{*}\left(x_{1}^{*}+2 \sqrt{m}\right)}{2 \sqrt{m}\left(m+x_{1}^{* 2}\right)}\left|e^{u_{1}(s)}-1\right|\right\} d s .
\end{aligned}
$$

By Lemma 2.2, we know that there exists a $T>0$ such that $x_{1}^{*} e^{u_{1}(t)}=x_{1}(t) \leq M_{1}$ for $t \geq T$. Hence for $t \geq T+\tau$, we have

$$
\begin{aligned}
D^{+} V_{11}(t) \leq & -x_{1}^{*}\left(a_{11}-\frac{a_{12} x_{2}^{*}\left(x_{1}^{*}+2 \sqrt{m}\right)}{2 \sqrt{m}\left(m+x_{1}^{* 2}\right)}\right)\left|e^{u_{1}(t)}-1\right|+\frac{a_{12} x_{2}^{*}}{2 \sqrt{m}}\left|e^{u_{2}(t)}-1\right| \\
& +a_{11} M_{1} \int_{t-\tau_{1}}^{t}\left\{a_{11} x_{1}^{*}\left|e^{u_{1}\left(s-\tau_{1}\right)}-1\right|+\frac{a_{12} x_{2}^{*}}{2 \sqrt{m}}\left|e^{u_{2}(s)}-1\right|\right. \\
& \left.+\frac{a_{12} x_{1}^{*} x_{2}^{*}\left(x_{1}^{*}+2 \sqrt{m}\right)}{2 \sqrt{m}\left(m+x_{1}^{* 2}\right)}\left|e^{u_{1}(s)}-1\right|\right\} d s .
\end{aligned}
$$

We now define a Lyapunov functional $V_{1}(t)$ as

$$
V_{1}(t)=V_{11}(t)+V_{12}(t)
$$

where

$$
\begin{aligned}
V_{12}(t)= & a_{11} M_{1} \int_{t-\tau_{1}}^{t} \int_{v}^{t}\left\{a_{11} x_{1}^{*}\left|e^{u_{1}\left(s-\tau_{1}\right)}-1\right|+\frac{a_{12} x_{2}^{*}}{2 \sqrt{m}}\left|e^{u_{2}(s)}-1\right|\right. \\
& \left.+\frac{a_{12} x_{1}^{*} x_{2}^{*}\left(x_{1}^{*}+2 \sqrt{m}\right)}{2 \sqrt{m}\left(m+x_{1}^{* 2}\right)}\left|e^{u_{1}(s)}-1\right|\right\} d s d v \\
& +a_{11}^{2} x_{1}^{*} M_{1} \tau_{1} \int_{t-\tau_{1}}^{t}\left|e^{u_{1}(s)}-1\right| d s .
\end{aligned}
$$

It then follows from (4.5)-(4.7) and (1.1) that for $t \geq T+\tau$

$$
\begin{aligned}
D^{+} V_{1}(t) \leq & -x_{1}^{*}\left[a_{11}-\frac{a_{12} x_{2}^{*}\left(x_{1}^{*}+2 \sqrt{m}\right)}{2 \sqrt{m}\left(m+x_{1}^{* 2}\right)}\right. \\
& \left.-a_{11} M_{1} \tau_{1}\left(a_{11}+\frac{a_{12} x_{2}^{*}\left(x_{1}^{*}+2 \sqrt{m}\right)}{2 \sqrt{m}\left(m+x_{1}^{* 2}\right)}\right)\right]\left|e^{u_{1}(t)}-1\right| \\
& +\frac{a_{12} x_{2}^{*}}{2 \sqrt{m}}\left(1+a_{11} M_{1} \tau_{1}\right)\left|e^{u_{2}(t)}-1\right| \\
\leq & -x_{1}^{*}\left[a_{11}-\left(\frac{a_{1}}{2 \sqrt{m}}+\frac{a_{1}}{x_{1}^{*}}-a_{11}\right)\right. \\
& \left.-a_{11} M_{1} \tau_{1}\left(a_{11}+\frac{a_{1}}{2 \sqrt{m}}+\frac{a_{1}}{x_{1}^{*}}-a_{11}\right)\right]\left|e^{u_{1}(t)}-1\right|
\end{aligned}
$$




$$
\begin{aligned}
& +\frac{a_{12} x_{2}^{*}}{2 \sqrt{m}}\left(1+a_{11} M_{1} \tau_{1}\right)\left|e^{u_{2}(t)}-1\right| \\
\leq & -x_{1}^{*}\left[2 a_{11}-\frac{a_{1}}{2 \sqrt{m}}-a_{1} \sqrt{\frac{a_{21}-a_{2}}{m a_{2}}}\right. \\
& \left.-a_{11} M_{1} \tau_{1}\left(\frac{a_{1}}{2 \sqrt{m}}+a_{1} \sqrt{\frac{a_{21}-a_{2}}{m a_{2}}}\right)\right]\left|e^{u_{1}(t)}-1\right| \\
& +\frac{a_{12} x_{2}^{*}}{2 \sqrt{m}}\left(1+a_{11} M_{1} \tau_{1}\right)\left|e^{u_{2}(t)}-1\right| \\
= & -r_{11} x_{1}^{*}\left|e^{u_{1}(t)}-1\right|-r_{12} x_{2}^{*}\left|e^{u_{2}(t)}-1\right| .
\end{aligned}
$$

The second equation of (4.2) can be rewritten as

$$
\begin{aligned}
\frac{d u_{2}}{d t}= & -a_{22} x_{2}^{*}\left(e^{u_{1}(t)}-1\right)+\frac{m a_{21} x_{1}^{*}\left(x_{1}\left(t-\tau_{2}\right)+x_{1}^{*}\right)}{\left(m+x_{1}^{2}\left(t-\tau_{2}\right)\right)\left(m+x_{1}^{* 2}\right)}\left(e^{u_{1}\left(t-\tau_{2}\right)}-1\right) \\
& +a_{22} x_{2}^{*} \int_{t-\tau_{3}}^{t} e^{u_{2}(s)} \frac{d u_{2}(s)}{d s} d s \\
= & -a_{22} x_{2}^{*}\left(e^{u_{1}(t)}-1\right)+\frac{m a_{21} x_{1}^{*}\left(x_{1}\left(t-\tau_{2}\right)+x_{1}^{*}\right)}{\left(m+x_{1}^{2}\left(t-\tau_{2}\right)\right)\left(m+x_{1}^{* 2}\right)}\left(e^{u_{1}\left(t-\tau_{2}\right)}-1\right) \\
& +a_{22} x_{2}^{*} \int_{t-\tau_{3}}^{t} e^{u_{2}(s)}\left\{\frac{m a_{21} x_{1}^{*}\left(x_{1}\left(s-\tau_{2}\right)+x_{1}^{*}\right)}{\left(m+x_{1}^{2}\left(s-\tau_{2}\right)\right)\left(m+x_{1}^{* 2}\right)}\left(e^{u_{1}\left(s-\tau_{2}\right)}-1\right)\right. \\
& \left.-a_{22} x_{2}^{*}\left(e^{u_{2}\left(s-\tau_{3}\right)}-1\right)\right\} d s .
\end{aligned}
$$

Let

$$
V_{21}(t)=\left|u_{2}(t)\right|
$$

Calculating the upper right derivative of $V_{21}(t)$ along solutions of (4.2), it follows from (4.9) and (4.10) that

$$
\begin{aligned}
D^{+} V_{21}(t) \leq & -a_{22} x_{2}^{*}\left|e^{u_{1}(t)}-1\right|+\frac{m a_{21} x_{1}^{*}\left(x_{1}\left(t-\tau_{2}\right)+x_{1}^{*}\right)}{\left(m+x_{1}^{2}\left(t-\tau_{2}\right)\right)\left(m+x_{1}^{* 2}\right)}\left|e^{u_{1}\left(t-\tau_{2}\right)}-1\right| \\
& +a_{22} x_{2}^{*} \int_{t-\tau_{3}}^{t} e^{u_{2}(s)}\left\{\frac{m a_{21} x_{1}^{*}\left(x_{1}\left(s-\tau_{2}\right)+x_{1}^{*}\right)}{\left(m+x_{1}^{2}\left(s-\tau_{2}\right)\right)\left(m+x_{1}^{* 2}\right)}\left|e^{u_{1}\left(s-\tau_{2}\right)}-1\right|\right. \\
& \left.+a_{22} x_{2}^{*}\left|e^{u_{2}\left(s-\tau_{3}\right)}-1\right|\right\} d s \\
\leq & -a_{22} x_{2}^{*}\left|e^{u_{1}(t)}-1\right|+\frac{a_{21}}{\sqrt{m}} x_{1}^{*}\left|e^{u_{1}\left(t-\tau_{2}\right)}-1\right| \\
& +a_{22} x_{2}^{*} \int_{t-\tau_{3}}^{t} e^{u_{2}(s)}\left\{\frac{a_{21}}{\sqrt{m}} x_{1}^{*}\left|e^{u_{1}\left(s-\tau_{2}\right)}-1\right|+a_{22} x_{2}^{*}\left|e^{u_{2}\left(s-\tau_{3}\right)}-1\right|\right\} d s .
\end{aligned}
$$


By Lemma 2.2, we see that there exists a $T>0$ such that $x_{2}^{*} e^{u_{2}(t)}=x_{2}(t) \leq M_{2}$ for $t \geq T$. Hence for $t \geq T+\tau$, we have

$$
\begin{aligned}
D^{+} V_{21}(t) \leq & -a_{22} x_{2}^{*}\left|e^{u_{1}(t)}-1\right|+\frac{a_{21}}{\sqrt{m}} x_{1}^{*}\left|e^{u_{1}\left(t-\tau_{2}\right)}-1\right| \\
& +a_{22} M_{2} \int_{t-\tau_{3}}^{t}\left\{\frac{a_{21}}{\sqrt{m}} x_{1}^{*}\left|e^{u_{1}\left(s-\tau_{2}\right)}-1\right|+a_{22} x_{2}^{*}\left|e^{u_{2}\left(s-\tau_{3}\right)}-1\right|\right\} d s .
\end{aligned}
$$

Define a Lyapunov functional $V_{2}(t)$ as

$$
V_{2}(t)=V_{21}(t)+V_{22}(t)
$$

where

$$
\begin{aligned}
V_{22}(t)= & a_{22} M_{2} \int_{t-\tau_{3}}^{t} \int_{v}^{t}\left\{\frac{a_{21}}{\sqrt{m}} x_{1}^{*}\left|e^{u_{1}\left(s-\tau_{2}\right)}-1\right|+a_{22} x_{2}^{*}\left|e^{u_{2}\left(s-\tau_{3}\right)}-1\right|\right\} d s d v \\
& +a_{22} M_{2} \tau_{3}\left\{\frac{a_{21}}{\sqrt{m}} x_{1}^{*} \int_{t-\tau_{2}}^{t}\left|e^{u_{1}(s)}-1\right| d s+a_{22} x_{2}^{*} \int_{t-\tau_{3}}^{t}\left|e^{u_{2}(s)}-1\right| d s\right\} \\
& +\frac{a_{21}}{\sqrt{m}} x_{1}^{*} \int_{t-\tau_{2}}^{t}\left|e^{u_{1}(s)}-1\right| d s .
\end{aligned}
$$

Then it follows from (4.11)-(4.13) that for $t \geq T+\tau$

$$
\begin{aligned}
D^{+} V_{2}(t) & \leq \frac{a_{21}}{\sqrt{m}}\left(1+a_{22} M_{2} \tau_{3}\right) x_{1}^{*}\left|e^{u_{1}(t)}-1\right|-a_{22} x_{2}^{*}\left(1-a_{22} M_{2} \tau_{3}\right)\left|e^{u_{2}(t)}-1\right| \\
& =-r_{21} x_{1}^{*}\left|e^{u_{1}(t)}-1\right|-r_{22} x_{2}^{*}\left|e^{u_{2}(t)}-1\right| .
\end{aligned}
$$

According to Assumptions (H5)-(H6), we know that $C=\left(r_{i j}\right)_{2 \times 2}$ is an $M$-matrix. Hence there exist positive constants $c_{i}(i=1,2)$ such that

$$
r_{11} c_{1}+r_{21} c_{2}=h_{1}>0 \text { and } r_{12} c_{1}+r_{22} c_{2}=h_{2}>0 .
$$

We now define a Lyapunov functional $V(t)$ as $V(t)=c_{1} V_{1}(t)+c_{2} V_{2}(t)$. Then we have from (4.8) and (4.14) that for $t \geq T+\tau$

$$
D^{+} V(t) \leq-h_{1} x_{1}^{*}\left|e^{u_{1}(t)}-1\right|-h_{2} x_{2}^{*}\left|e^{u_{2}(t)}-1\right| .
$$

Since system (1.1) is uniformly persistent, one can see that there exist positive constants $m_{k}(k=1,2)$ and a $T^{*} \geq T+\tau$ such that $x_{k}^{*} e^{u_{k}(t)}=x_{k}(t) \geq m_{k}(k=1,2)$ for $t \geq T^{*}$. Using the mean value theorem one obtains

$$
x_{k}^{*}\left|e^{u_{k}(t)}-1\right|=x_{k}^{*} e^{\theta_{k}(t)}\left|u_{k}(t)\right| \geq m_{k}\left|u_{k}(t)\right| \quad(k=1,2),
$$


where $x_{k}^{*} e^{\theta_{k}(t)}$ lies between $x_{k}(t)$ and $x_{k}^{*}$. Let $\delta=\min \left\{m_{1} h_{1}, m_{2} h_{2}\right\}$. Then it follows from (4.15) that for $t \geq T^{*}$

$$
D^{+} V(t) \leq-\delta\left(\left|u_{1}(t)\right|+\left|u_{2}(t)\right|\right)
$$

Noting that $V(t) \geq \min \left\{c_{1}, c_{2}\right\}\left(\left|u_{1}(t)\right|+\left|u_{2}(t)\right|\right)$, we can conclude from the Lyapunov theorem and (4.16) that the zero solution of (4.2) is globally asymptotically stable and hence that the positive equilibrium $E^{*}\left(x_{1}^{*}, x_{2}^{*}\right)$ of $(1.1)$ is globally asymptotically stable.

COROllaRY 4.1. Let $(\mathrm{H} 1)-(\mathrm{H} 2)$ hold. Assume further that

$$
a_{22}\left(2 a_{11}-\frac{a_{1}}{2 \sqrt{m}}-a_{1} \sqrt{\frac{a_{21}-a_{2}}{m a_{2}}}\right)-\frac{a_{12} a_{21}}{2 m}>0 .
$$

Then the positive equilibrium $E^{*}\left(x_{1}^{*}, x_{2}^{*}\right)$ of system (1.1) is globally asymptotically stable provided that $0 \leq \max \left\{\tau_{1} e^{a_{1} \tau_{1}}, \tau_{3} e^{a_{21} \tau_{3}}\right\} \leq \tau_{G}$, where

$$
\begin{aligned}
\tau_{G}= & \frac{1}{2\left(B_{1} B_{4}-B_{2} B_{3}\right)}\left[A_{1} B_{4}+A_{4} B_{1}+A_{2} B_{3}+A_{3} B_{2}\right. \\
& -\left(\left(A_{1} B_{4}+A_{4} B_{1}+A_{2} B_{3}+A_{3} B_{2}\right)^{2}\right. \\
& \left.\left.-4\left(A_{1} A_{4}-A_{2} A_{3}\right)\left(B_{1} B_{4}-B_{2} B_{3}\right)\right)^{1 / 2}\right]
\end{aligned}
$$

in which

$$
\begin{aligned}
& A_{1}=2 a_{11}-\frac{a_{1}}{2 \sqrt{m}}-a_{1} \sqrt{\frac{a_{21}-a_{2}}{m a_{2}}}, \quad A_{2}=\frac{a_{12}}{2 \sqrt{m}}, \quad A_{3}=\frac{a_{21}}{\sqrt{m}}, \quad A_{4}=a_{22}, \\
& B_{1}=a_{1}^{2}\left(\frac{1}{2 \sqrt{m}}+\sqrt{\frac{a_{21}-a_{2}}{m a_{2}}}\right), \quad B_{2}=\frac{a_{1} a_{12}}{2 \sqrt{m}}, \quad B_{3}=\frac{a_{21}^{2}}{\sqrt{m}}, \quad B_{4}=a_{21} a_{22} \text {. }
\end{aligned}
$$

\section{Examples}

If the instantaneous system related to (1.1) has a globally stable equilibrium $E^{*}$, then, in general, it is anticipated that $E^{*}$ is globally stable for $\max \left\{\tau_{1}, \tau_{3}\right\}<\tau_{G}$, and locally asymptotically stable for $\max \left\{\tau_{1}, \tau_{3}\right\}<\tau_{L}$, where $0<\tau_{G}<\tau_{L}$. From the results derived in previous sections, it is not easy to determine whether, in general, the sufficient conditions given there ensure the ordering of the values of $\tau$ given above. However, it is straightforward to find specific examples for which this ordering holds as we now demonstrate. 


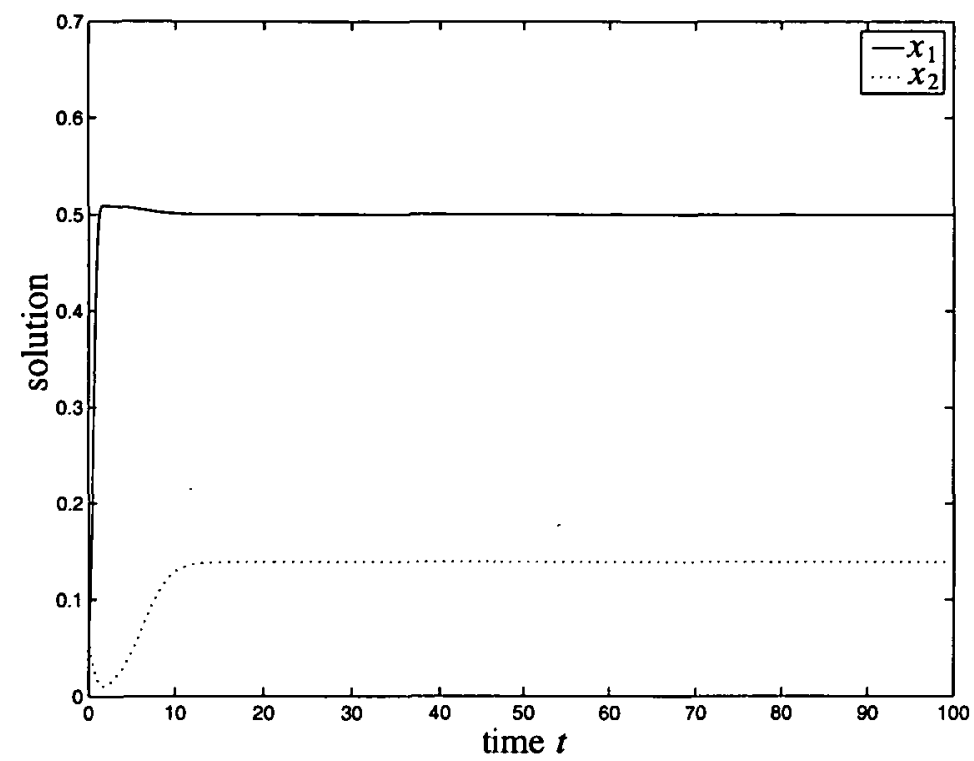

FIGURE 1. The temporal solution found by numerical simulation of system (5.1) with $\tau_{1}=0.1382, \tau_{2}=1$ and $\tau_{3}=0.1382$. Initial data are $\left(x_{1}, x_{2}\right) \equiv(0.06,0.06)$.

EXAMPLE 1. Consider the following system:

$$
\begin{aligned}
& \dot{x}_{1}(t)=x_{1}(t)\left(3-\frac{53}{9} x_{1}\left(t-\tau_{1}\right)-\frac{x_{1}(t) x_{2}(t)}{1+x_{1}^{2}(t)}\right), \\
& \dot{x}_{2}(t)=x_{2}(t)\left(-1+\frac{70}{9} \frac{x_{1}^{2}\left(t-\tau_{2}\right)}{1+x_{1}^{2}\left(t-\tau_{2}\right)}-4 x_{2}\left(t-\tau_{3}\right)\right) .
\end{aligned}
$$

System (5.1) has a unique positive equilibrium $E^{*}(1 / 2,5 / 36)$. Using Theorem 2.1 we know that system (5.1) is uniformly persistent provided that $\tau_{1} \leq 0.5$. By Theorem 3.1 and Corollary 3.1 , we see that the positive equilibrium $E^{*}(1 / 2,5 / 36)$ is locally asymptotically stable provided that $\max \left\{\tau_{1}, \tau_{3}\right\}<0.1382$, or $132129 \tau_{1}+2800 \tau_{3}<$ 36918 and $4293 \tau_{1}+7300 \tau_{3}<1602$. By Theorem 4.1 and Corollary 4.1, we know that the positive equilibrium $E^{*}(1 / 2,5 / 36)$ of $(5.1)$ is globally asymptotically stable provided that $\max \left\{\tau_{1}, \tau_{3}\right\}<0.02384$, or $r_{11}>0, r_{22}>0$ and $r_{11} r_{22}-r_{12} r_{21}>0$, where $r_{11}=185 / 18-\sqrt{61}-(53 / 9) M_{1} \tau_{1}(3 / 2+\sqrt{61}), r_{12}=-\left(1+53 M_{1} \tau_{1} / 9\right) / 2$, $r_{21}=-70\left(1+4 M_{2} \tau_{3}\right) / 9, r_{22}=4\left(1-4 M_{2} \tau_{3}\right), M_{1}=27 e^{3 \tau_{1}} / 53, M_{2}=35 e^{90 \tau_{3} / 7} / 18$.

Using L. F. Shampine and S. Thompson's program dde23 for solving DDEs [35], numerical simulation shows that if $\max \left(\tau_{1}, \tau_{3}\right\}<0.1382$, the positive equilibrium $E^{*}$ is locally stable (see Figure 1). In fact, after testing a large range of initial data, it seems that $E^{*}$ is also globally stable for $\max \left\{\tau_{1}, \tau_{3}\right\}<0.1382$ and further numerical simulations suggest that it remains so for $\max \left\{\tau_{1}, \tau_{3}\right\}<0.43$ (see Figure 2). For 


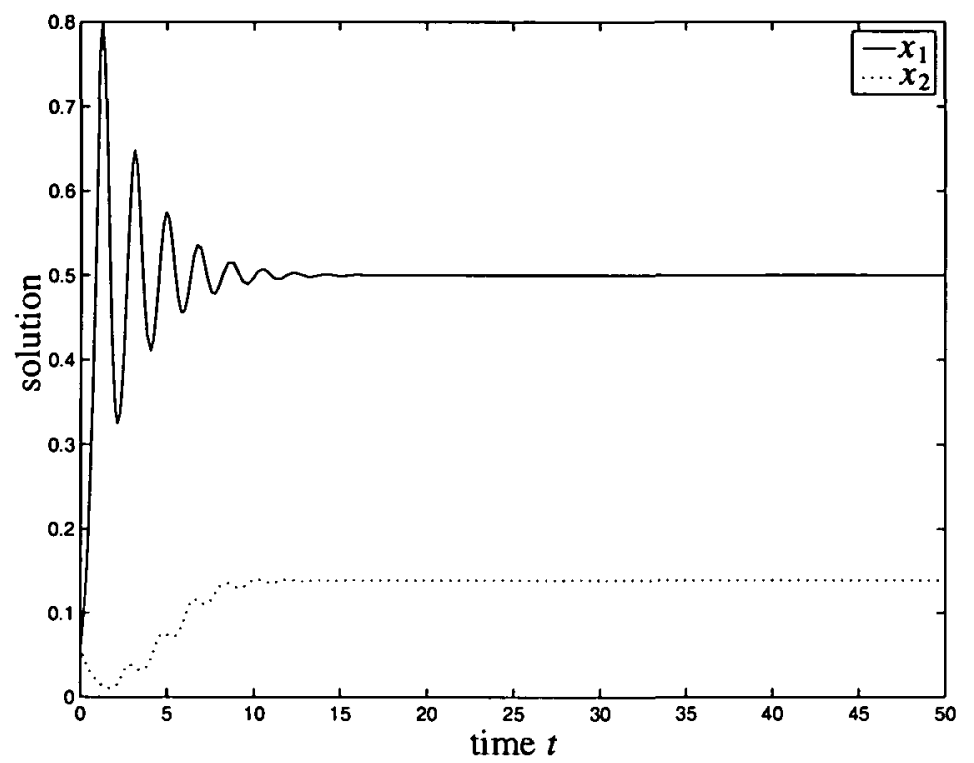

FIGURE 2. The temporal solution found by numerical simulation of system (5.1) with $\tau_{1}=0.43, \tau_{2}=1$ and $\tau_{3}=0.43$. Initial data are $\left(x_{1}, x_{2}\right) \equiv(0.06,0.06)$.

$\min \left\{\tau_{1}, \tau_{3}\right\}>0.5$, numerical simulation shows that a stability switch occurs and the positive equilibrium $E^{*}$ becomes unstable (see Figure 3 ). These results suggest that the bounds derived in previous sections are somewhat conservative.

EXAMPLE 2. Consider another delayed system:

$$
\begin{aligned}
& \dot{x}_{1}(t)=x_{1}(t)\left(2-0.1 x_{1}(t)-\frac{x_{1}(t) x_{2}(t)}{1+x_{1}^{2}(t)}\right), \\
& \dot{x}_{2}(t)=x_{2}(t)\left(-1+4 \frac{x_{1}^{2}\left(t-\tau_{2}\right)}{1+x_{1}^{2}\left(t-\tau_{2}\right)}-0.1 x_{2}(t)\right) .
\end{aligned}
$$

System (5.2) has a unique positive equilibrium $E^{*}(0.7353,4.0372)$. By Theorem 2.1 we see that system (5.2) is uniformly persistent. It is easy to calculate that

$$
A_{11}+B_{11}=-0.6480, \quad A_{22}\left(A_{11}+B_{11}\right)+A_{12} A_{21}=-3.2491 .
$$

Then the positive equilibrium of the corresponding instantaneous system $\left(\tau_{2}=0\right)$ of (5.2) is locally asymptotically stable. From the discussions in Section 3 , we see that as $\tau_{2}$ increases, a stability switch occurs. Thus there is a positive constant $\tau_{0}$ such that for $\tau_{2}>\tau_{0}, E^{*}$ becomes unstable. Numerical simulation confirms our above observation at $\tau_{2}=0.35$ (see Figure 4). On the other hand, it is easy to show that (4.17) doesn't 


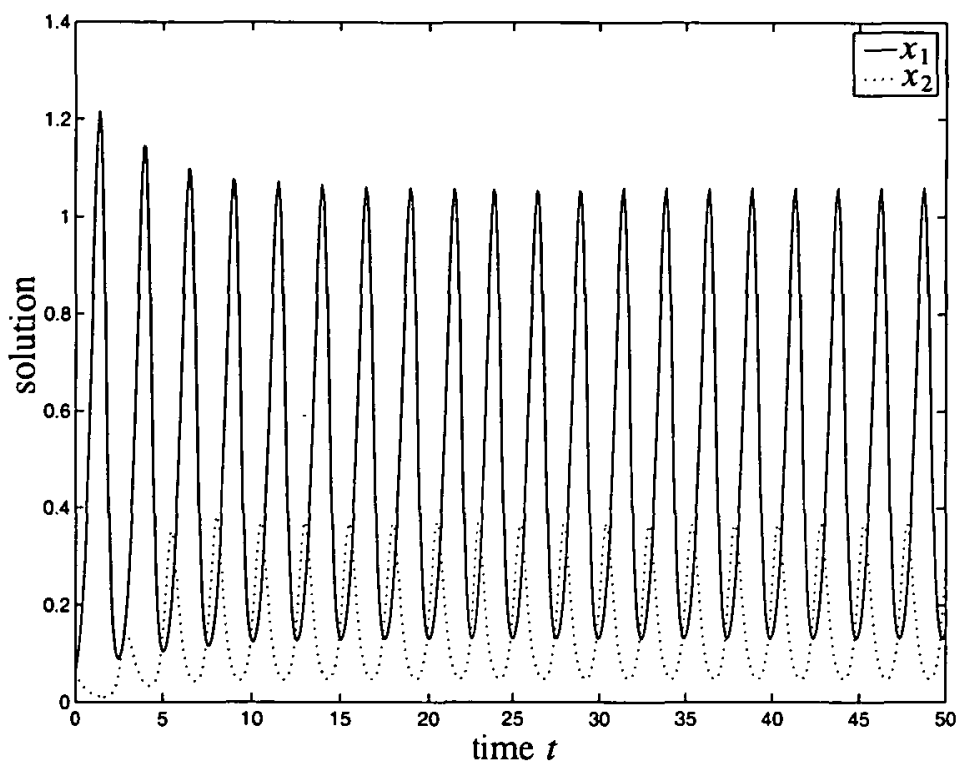

FIGURE 3. The temporal solution found by numerical simulation of system (5.1) with $\tau_{1}=0.6, \tau_{2}=1$ and $\tau_{3}=0.6$. Initial data are $\left(x_{1}, x_{2}\right) \equiv(0.06,0.06)$.

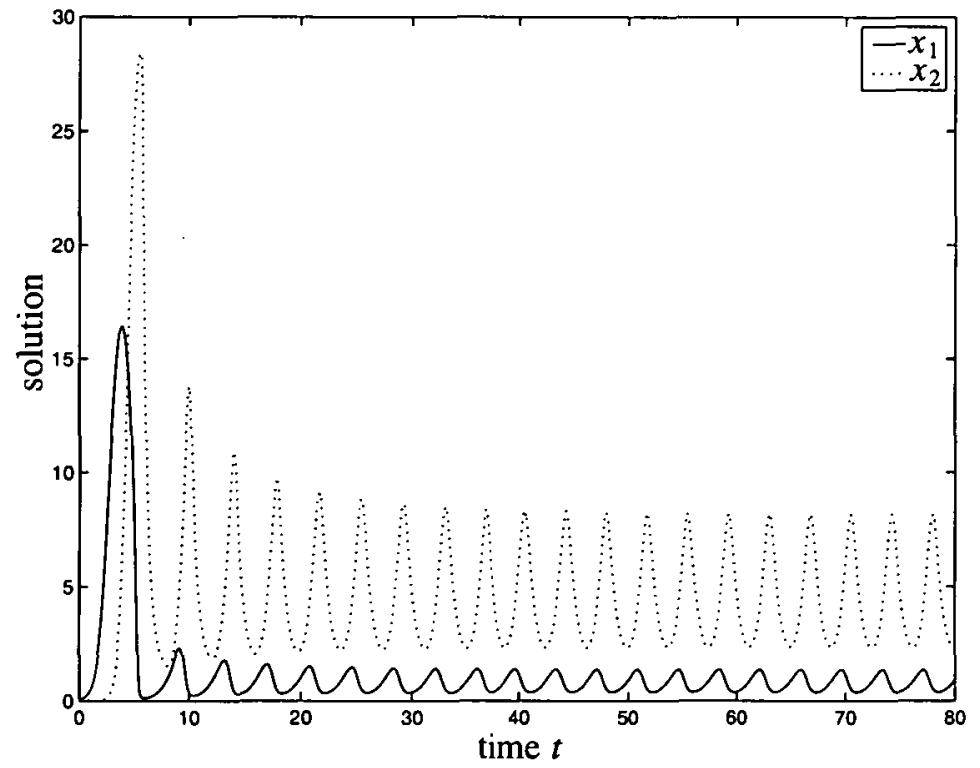

FIGURE 4. The temporal solution found by numerical simulation of system (5.2) with $\tau_{2}=0.35$. Initial data are $\left(x_{1}, x_{2}\right) \equiv(0.06,0.06)$. 


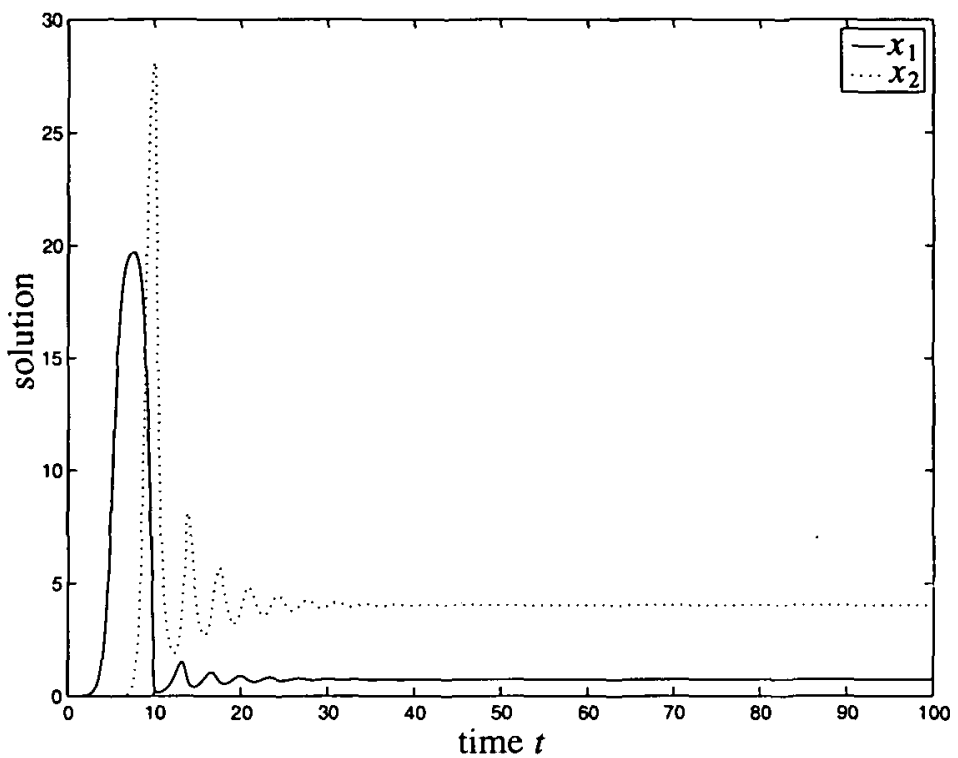

FIGURE 5. The temporal solution found by numerical simulation of system (5.2) with $\tau_{2}=0.2$. Initial data are $\left(x_{1}(\theta), x_{2}(\theta)\right) \equiv(0.0006,0.0006)$.

hold for system (5.2). After testing a large range of initial data, it appears that $E^{*}$ is locally and globally asymptotically stable for $\tau_{2}<0.2$ (see Figure 5).

\section{Discussion}

In this paper, we investigated the global dynamics of a predator-prey model with Holling type III functional response and time delays. Borrowing the result of Freedman and Ruan [7], we have established sufficient conditions for system (1.1) to be uniformly persistent. By means of suitable Lyapunov functionals, we have discussed the local and global asymptotic stability of a positive equilibrium of system (1.1). By Theorem 2.1, we see that system (1.1) with initial conditions (1.2) will be uniformly persistent if the delay due to negative feedback of the prey is small enough, and the intrinsic growth rate of the prey species and the conversion rate of the predator are high and the death rate of the predator and the intra-specific competition rate of the prey are low. By Theorems 3.1 and 4.1, we have shown that under some conditions, if the positive equilibrium of the corresponding instantaneous system is locally and globally stable, then local and global stability of the positive equilibrium of the delayed system (1.1) will persist when time delays due to negative feedbacks of the prey and predator are sufficiently small. 
We would like to mention here that numerical simulations in Examples 1 and 2 show that our results in Theorems 3.1 and 4.1 have room for improvement. We leave this for future work.

\section{Acknowledgements}

The authors would like to extend their appreciation to the anonymous referees for their many helpful comments and suggestions which greatly improved the presentation of this paper. The first author wishes to thank the Department of Mathematics, University of Dundee for the hospitality, support and the excellent working conditions provided to him during his visit to Dundee.

\section{References}

[1] K. M. Bailey, "Predation on juvenile flatfish and recruitment variability", Neth. J. Sea Res. 32 (1994) 175-189.

[2] A. D. Bazykin, Structural and dynamic stability of model predator-prey systems (Int. Inst. Appl. Syst. Analysis, Laxenburg, 1976).

[3] A. D. Bazykin, F. S. Berezovskaya, G. A. Denisov and Yu A. Kuznetzov, "The influence of predator saturation effect and competition among predators on predator-prey system dynamics", Ecol. Model. 14 (1981) 39-57.

[4] E. Beretta and Y. Kuang, "Convergence results in a well-known delayed predator-prey system", $J$. Math. Anal. Appl. 204 (1996) 840-853.

[5] J. M. Cushing, Integro-differential equations and delay models in population dynamics (Springer, Heidelberg, 1977).

[6] H. I. Freedman, Deterministic mathematical models in population ecology, Monogr. Textbooks Pure Appl. Math. 57 (Marcel Dekker, New York, 1980).

[7] H. I. Freedman and S. Ruan, "Uniform persistence in functional differential equations", J. Differential Equations 115 (1995) 173-192.

[8] K. Gopalsamy, "Harmless delay in model systems", Bull. Math. Biol. 45 (1983) 295-309.

[9] K. Gopalsamy, "Delayed responses and stability in two-species systems", J. Austral. Math. Soc. Ser. B 25 (1984) 473-500.

[10] K. Gopalsamy, Stability and oscillations in delay differential equations of population dynamics (Kluwer, Dordrecht/Norwell, MA, 1992).

[11] J. Hainzl, "Stability and Hopf bifurcation in a predator-prey system with several parameters", SIAM J. Appl. Math. 48 (1988) 170-190.

[12] M. P. Hassell (Ed.), The dynamics of arthropod predator-prey systems (Princeton University Press, Princeton, NJ, 1978) 237.

[13] A. Hastings, "Delays in recruitment at different trophic levels: effects on stability", J. Math. Biol. 21 (1984) 35-44.

[14] X. Z. He, "Stability and delays in a predator-prey system", J. Math. Anal. Appl. 198 (1996) 355-370.

[15] X. Z. He, "Degenerate Lyapunov functionals of a well-known predator-prey model with discrete delays", Proc. Roy. Soc. Edinburgh Sect. A 129 (1999) 755-771. 
[16] M. Hesaaraki and S. M. Moghadas, "Existence of limit cycles for predator-prey systems with a class of functional responses", Ecol. Model. 142 (2001) 1-9.

[17] M. Hixon and M. Carr, "Synergistic predation, density dependence and population regulation in marine fish", Science 277 (1997) 946-949.

[18] J. Hofbauer and K. Sigmund, The theory of evolution and dynamical systems (Cambridge University Press, New York, 1988).

[19] C. S. Holling, "The components of predation as revealed by a study of small mammal predation of the European pine sawfly", Can. Entomol. 91 (1959) 293-320.

[20] C. S. Holling, "Some characteristics of simple types of predation and parasitism", Can. Entomol. 91 (1959) 385-398.

[21] C. S. Holling, "The functional response of predators to prey density and its role in mimicry and population regulation", Mem. Entomolog. Soc. Can. 45 (1965) 3-60.

[22] V. Hutson, "The existence of an equilibrium for permanent systems", Rocky Mountain J. Math. 20 (1990) 1033-1040.

[23] N. D. Kazarinoff and P. Van Den Driessche, "A model predator-prey system with functional response", Math. Biosci. 39 (1978) 125-134.

[24] R. E. Kooij and A. Zegeling, "Qualitative properties of two-dimensional predator-prey systems", Nonlinear Anal. 29 (1997) 693-715.

[25] Y. Kuang, Delay differential equations with applications in population dynamics (Academic Press, New York, 1993).

[26] Y. Kuang, "Global stability in delay differential systems without dominating instantaneous negative feedbacks", J. Differential Equations 119 (1995) 503-532.

[27] Y. Kuang and H. I. Freedman, "Uniqueness of limit cycles in Gauss-type models of predator-prey systems", Math. Biosci. 88 (1988) 67-84.

[28] N. MacDonald, Time lags in biological models (Springer, Heidelberg, 1978).

[29] R. M. May, "Limit cycles in predator-prey communities", Science 177 (1972) 900-902.

[30] R. M. May, "Time delay versus stability in population models with two and three trophic levels", Ecology 4 (1973) 315-325.

[31] W. W. Murdoch, "Switching in general predators: experiments on predator specificity and stability of prey populations", Ecol. Monogr. 39 (1969) 335-354.

[32] W. W. Murdoch, S. Avery and M. E. B. Smyth, "Switching in predatory fish", Ecology 56 (1975) $1094-1105$.

[33] W. W. Murdoch and R. J. Marks, "Predation by coccinellid beetles: experiments on switching", Ecology 54 (1973) 160-167.

[34] S. Ruan, "Absolute stability, conditional stability and bifurcation in Kolmogorov-type predatorprey systems with discrete delays", Quart. Appl. Math. 59 (2001) 159-173.

[35] L. F. Shampine and S. Thompson, "Solving DDEs in MATLAB", Appl. Numer. Math. 37 (2002) 441-458.

[36] J. Sugie, R. Kohno and R. Miyazaki, "On a predator-prey system of Holling type", Proc. Amer. Math. Soc. 125 (1997) 2041-2050.

[37] W. Wang and Z. Ma, "Harmless delays for uniform persistence", J. Math. Anal. Appl. 158 (1991) 256-268.

[38] E. M. Wright, “A nonlinear differential difference equation", J. Reine Angew. Math. 194 (1955) 66-87.

[39] R. Xu and P. Yang, "Persistence and stability in a three species food-chain system with time delays", in Proceedings of the International Conference on Mathematical Biology, Advanced Topics in Biomathematics (ed. L. S. Chen et al.), (World Scientific, Singapore, 1998) 277-283. 